\title{
Group, One-on-One, or Internet? Preferences for Mindfulness Meditation Delivery Format and their Predictors
}

\author{
Helané Wahbeh" ${ }^{*}, 1,2$, Matthew N. Svalina ${ }^{1}$ and Barry S. Oken ${ }^{1}$ \\ ${ }^{I}$ Oregon Health \& Science University, Portland, Oregon, USA \\ ${ }^{2}$ National College of Natural Medicine, Portland, Oregon, USA
}

\begin{abstract}
Objectives: Group mindfulness meditation interventions have improved symptoms in many health conditions. However, many people are unwilling to receive group treatment, so alternative delivery methods such as individual and internet may be a useful option. The study objective was to examine mindfulness meditation intervention delivery format preferences and their relationship to potential predictors.

Design: An online survey was conducted of adult English speakers. Data was collected on interest and preference for internet, individual, or group formats of a mindfulness meditation intervention. Age, gender, personality, and posttraumatic stress disorder score and status and depression status were also collected.

Results and Conclusion: 500 eligible participants completed the survey (mean age 39 \pm 15 ; range 18-70; 68\% female). Participants were more interested in the Internet $(n=356)$ and individual formats $(n=384)$ than the group format $(n=245)$. Fifty-five participants (11\%) said they would refuse a group format. Internet was the first choice format for most participants (Internet 212 (43\%), Individual 187 (38\%), Group $97(20 \%)$ and group was the last choice for most participants (Internet 140 (29\%), Individual 70 (14\%), Group 279 (57\%)). Age, extraversion and emotional stability were significant in predicting first choice format. These results support the need for more research and implementation of alternative mindfulness meditation intervention delivery formats. Future research will incorporate additional predictors and include a broader range of participants.
\end{abstract}

Keywords: Complementary and alternative medicine, group, internet, individual, mind-body medicine, mindfulness meditation.

\section{INTRODUCTION}

Group mindfulness meditation interventions (MMI) improve a variety of health conditions, are inexpensive, easy to implement, have minimal side effects, and engage patients to take an active role in their treatment [1-10]. MMI consistently improves a spectrum of "mental health measures such as quality of life, depression, anxiety, coping style," and affective dimensions of disability, as reported in a metaanalysis of 64 studies of varied health conditions [7]. Since this meta-analysis, similar improvements have been found in anxiety [11, 12], sleep disturbances [13], stress [14], and chronic pain [15]. Group mindfulness-based formats have many benefits. The group format usually costs less than individual therapy because one therapist can see many patients in a session. Groups can also provide motivation and synergistic learning opportunities for the participants. Meeting other people with similar or other issues can give the participants a wider perspective on their own situation and allow them to see how others handle their problems. Participants can provide encouragement and emotional support for each other instilling a sense of camaraderie [16].

*Address correspondence to this author at the Oregon Health \& Science University, 3181 SW Sam Jackson Park Rd. CR120, Portland, Oregon 97239, USA; Tel: 503-494-3528; Fax: 503-494-9520;

E-mail:wahbehh@ohsu.edu
However, the group format may also be an obstacle for people who are interested in MMI.

The group format could be problematic for some participants, because it requires people to share in public, attend at a specific time and day, and travel to a specific location. These requirements are barriers for people to attend group MMI. Sharing in public is aversive to many people especially those with sensitive diagnoses like posttraumatic stress disorder (PTSD) or depression. While MMI may help improve PTSD symptoms [17, 18], the group format could be problematic for people with PTSD because they prefer individual therapy and are less willing to attend group sessions [19]. Attrition rates are as high as $50 \%$ for people with PTSD in group MMI [20]. This is true for other sensitive diagnoses as well [21]. These patients may prefer alternative formats of therapy that are convenient and private and avoid disclosure of personal issues in a group setting. Many people who are interested in MMI, such as parents or other caregivers, have busy schedules and adding a 2.5 hour weekly class and a full day retreat is prohibitive. Travelling to group classes can be challenging for those who live far from the location. Others for whom local groups are not available, such as rural residents, may also benefit from nongroup formats [22]. Developing an alternative delivery format for this highly effective intervention is essential. 
While alternative formats for other psychotherapeutics such as cognitive behavioral therapy have been established as viable and effective, especially for PTSD and anxiety disorders [23], internet and individual versions of MMI are just beginning to be examined. Online MMI programs have been evaluated for depression [24, 25], irritable bowel syndrome [26, 27], stress reduction [28], and pain [29] with some positive preliminary effects. Brief mindfulness meditation programs have also been evaluated in the workplace [30, 31]. Individual format $\mathrm{MMI}$ has been reported in a case study for problem gambling [32] and our own lab has developed a standardized individual MMI protocol with positive preliminary findings in stressed older adults [10]. A recent systematic review and meta-analysis evaluated the feasibility, cost-effectiveness and benefits of self-help and alternative format guided mindfulness meditation intervention studies [33]. They reported increased mindfulness and acceptance and decreased depression and anxiety symptoms. However, only four of the fifteen included studies were MMI specific [33]. While these studies demonstrate the feasibility of delivering a self-help intervention outside of the traditional group or therapist format, further studies are needed to evaluate the efficacy of alternative delivery formats.

Before such research is conducted, it is necessary to evaluate the perceived need for alternative formats and to examine predictors that play a role in determining preferences for format type. To our knowledge, predictors of delivery format preferences have not been formally examined. This cross-sectional study's purpose was to evaluate the perceived need for alternative formats of internet and individual MMI compared to the standard group format by assessing format preferences. In addition, the predictors that may mediate this preference were also examined (age, gender, PTSD symptoms and status, depression status, and personality characteristics). We hypothesized that more people would prefer the internet and individual formats compared to group as their first choice format. In addition, we hypothesized that the choice would be mediated by age with younger participants choosing internet over group, extraversion with more extraverted individuals choosing group over internet and individual, and those with more PTSD symptoms choosing internet and individual over group.

\section{MATERIALS AND METHODOLOGY}

We conducted a cross-sectional online survey between February 2012 and February 2014. Inclusion criteria were adults aged 18-70 years with the ability to understand and provide informed consent in English. There were no exclusion criteria. The survey was created and distributed using the SurveyMonkey software online (www.surveymo nkey.com). Participants were recruited via social networking, bulletin board, and aggregation websites through online advertisements throughout the United States. Individuals diagnosed with PTSD were over-sampled by sending a personal invitation to 50 past participants who participated in previous research studies. Ads were also posted on depression and PTSD listservs. After giving informed consent through the survey, participants completed a selfadministered survey. The Institutional Review Board of the
Oregon Health \& Science University approved online advertisements, survey questionnaire and format, and data collection protocols.

\section{Measures}

Online survey- The survey was composed of seven main questions and took approximately 5-10 minutes to complete. The survey began by stating "Imagine that you will participate in a research study on three different mindfulness therapy formats. All formats will present the same material. All formats will have a one-hour session each week for six weeks." Question 1 was: "How interested would you be in the following formats? Internet format which includes visiting a website from any location and reviewing the material online; Individual format which includes visiting the clinic and reviewing the material with a teacher one-onone; Group format which includes visiting the clinic and reviewing the material with a teacher in a group session with other participants." Answer choices included a 5-point Likert scale (Would refuse to take this version, Doesn't interest me, Neutral, Interests me, and Very interested in this version). Rating the internet, individual, and group format in this question was mandatory. There was also an optional text box asking "Why or why not would you be interested in taking each of these formats?" Question 2 was "Now if you had to make one choice... which would be your first choice (Internet, Individual or Group).... which would be your last choice (Internet, Individual or Group). Question 3 was a brief PTSD symptom checklist [34]. Question 4 was a depression screen [35]. Question 5 asked about age. Question 6 asked about gender. Question 7 was a 10-item personality inventory [36]. The PTSD and depression screenings were included because while we recruited from the general population, we were interested in the prevalence in the population sampled of PTSD symptoms and status and depression status and their relationship to format preferences. The personality inventory was included to assess personality as a potential predictor of format preference.

Qualitative analysis- Participants were given the option of expounding upon why they would or would not choose a particular delivery format in a brief expository text box and could make comments on multiple formats. Each transcript was read several times to gain a strong, overall sense of the data [37]. Using an inductive coding approach, descriptive parent and child codes were created and included in a codebook that had the definition of each code and a brief description of when to use it (Table 1) [38]. Each excerpt was coded and checked using the Dedoose web-based qualitative data analysis software for managing, analyzing, and presentation qualitative and mixed method research data (Dedoose, 4.1, Sociocultural Research Consultants, LLC., Los Angeles, CA).

PTSD screening- $A$ six-item abbreviated PTSD Checklist was administered to evaluate PTSD symptoms and assess PTSD status. This instrument takes six-items from the gold standard 17 item PTSD Checklist [39]. The abbreviated version has been validated against the full version using a 14 point cutoff for a positive abbreviated test (versus a 30 point cutoff used in the full version). The abbreviated version has a sensitivity of $0.92 \pm 0.19$ and a specificity of $0.72 \pm 0.06$. 
Table 1. Characteristics of respondents by first choice format preference.

\begin{tabular}{|l|c|c|c|c|}
\hline & Internet (n= 212) & Individual (n= 187) & Group (n= 97) & Statistics* \\
\hline \hline Age & $39 \pm 15$ & $37 \pm 17$ & $42 \pm 14$ & $X^{2}=7.99 p=0.02$ \\
\hline Women & $138(65 \%)$ & $127(67 \%)$ & $70(72 \%)$ & $X^{2}=1.5 p=0.46$ \\
\hline PCL Score & $17.2 \pm 6.2$ & $16.8 \pm 5.9$ & $18.0 \pm 6.0$ & $X^{2}=1.8 p=0.40$ \\
\hline Depression status + & $154(73 \%)$ & $142(76 \%)$ & $81(83 \%)$ & $X^{2}=4.3 p=0.12$ \\
\hline PTSD status + & $143(67 \%)$ & $136(73 \%)$ & $70(72 \%)$ & $X^{2}=1.5 p=0.50$ \\
\hline PTSD/Dep + & $136(64 \%)$ & $120(64 \%)$ & $66(68 \%)$ & $X^{2}=0.52 p=0.77$ \\
\hline Extraversion & $3.4 \pm 1.7$ & $3.5 \pm 1.7$ & $3.9 \pm 1.8$ & $X^{2}=3.6 p=0.16$ \\
\hline Agreeableness & $4.9 \pm 1.4$ & $4.8 \pm 1.5$ & $4.9 \pm 1.3$ & $X^{2}=0.04 p=0.98$ \\
\hline Conscientiousness & $4.8 \pm 1.6$ & $4.7 \pm 1.7$ & $4.6 \pm 1.7$ & $X^{2}=1.6 p=0.44$ \\
\hline Emotional Stability & $3.9 \pm 1.7$ & $4.0 \pm 1.7$ & $3.6 \pm 1.5$ & $X^{2}=2.9 p=0.24$ \\
\hline Openness to Experience & $5.1 \pm 1.5$ & $5.1 \pm 1.7$ & $5.3 \pm 1.4$ & $X^{2}=0.81 p=0.67$ \\
\hline
\end{tabular}

Values for continuous variables are listed as mean plus or minus standard deviation. Categorical variables are listed as number and percentage of respondents. PCL- PTSD Checklist; +- positive screen. For PTSD status, a score $>14$ was considered a positive screen. For Depression status, answering Yes to either of the two screening questions was considered a positive screen.

*Kruskal-Wallis test for continuous variables; Pearson chi-square test for categorical variables.

This has been found to be adequate for screening purposes [34].

Depression screening- Two questions were included from the "Primary Care Evaluation of Mental Disorders patient questionnaire: 1) During the past month, have you often been bothered by feeling down, depressed, or hopeless? and 2) During the past month, have you often been bothered by little interest or pleasure in doing things? A "Yes" response to either of these questions was considered a positive test." This brief instrument has been evaluated as a highly sensitive $96 \%$ (95\% confidence interval [CI], 90$99 \%$ ) and specific 57\% (95\% CI 53-62\%) screening tool for depression and validated against gold-standard instruments [35].

Personality Inventory- The Ten-item Personality Inventory (TIPI) asks whether the user sees himself as 10 different personality characteristics. The user then rates their agreement to each characteristic on a 7-point Likert scale (Disagree strongly, Disagree moderately, Disagree a little, Neither agree nor disagree, Agree a little, Agree moderately, Agree strongly). Scores are then tabulated for extraversion, agreeableness, conscientiousness, emotional stability, and openness to experience. The inventory reaches adequate psychometric levels in terms of: (a) convergence with widely used gold standard measures in self, observer, and peer reports, (b) test-retest reliability, (c) patterns of predicted external correlates, and (d) convergence between self and observer ratings [36]. The five factor model of personality is a robust and well-studied paradigm composed of five generally accepted foundational personality parameters: extraversion, agreeableness, conscientiousness, emotional stability and neuroticism, and openness to experience. Though the precise definitions of the five factors have been debated, they are generally accepted to represent; socially adaptable $v s$ solitary and reserved (extraversion), hostile $v s$ friendly (agreeableness), interpretation and evaluation of individual choices vs carelessness in action (conscientiousness), balance and control of emotions vs anxiety or neuroticism (emotional stability), and intelligence and flexibility of thought (openness to experience) [40].

\section{Statistical Analysis}

Data was first described qualitatively for the entire sample. Then potential predictors were qualitatively described by first format preference choice. Continuous data was evaluated for normality and found to be non-normally distributed. Differences between potential predictors by first format preference choice were analyzed with Kruskal-Wallis one-way analysis of variance for non-parametric continuous data and Pearson chi-square test for categorical variables. Any difference between the distributions of interest by format was assessed with a Pearson chi-square test for categorical variables (Interest, Format). Multinomial logistic regression was used to identify whether first and last choice format were associated with predictors. First choice and last choice were used as the categorical dependent variable in two multinomial logistic regression analyses. Multinomial logistic regression was used because the dependent variable (First/Last) has more than two categories (Internet, Individual, and Group). Group format was the reference format with internet and individual formats evaluated relative to the group format. Potential predictors in the model were Age, Gender ( $1=$ male, $2=$ female), Depression Status $(0=$ no, $1=y e s)$, PTSD Checklist Score, and Personality Categories (extraversion, agreeableness, conscientiousness, emotional stability, openness). Variables with probabilities at a level of 0.10 or higher were removed from the analysis. Relative risk ratios were then calculated for greater ease of interpretation. All statistics were conducted in Stata 12.0 (Statacorp, LP, USA).

Missing data was handled in the following manner. TIPI had 35 randomly missing response items out of the 5000 possible $(0.7 \%)$. Missing items were replaced with a "Neither agree nor disagree response" to ensure proper calculation of characteristics. The PCL Checklist had 10 randomly missing response items out of the 3000 possible 
$(0.3 \%)$. Missing items were replaced with the average of the other PCL items for that individual to allow for a PCL total score. Question 1 on format interest had missing items (internet-4; individual-3, group-7) as did Question 2 on First choice (4) and Last choice (11). Since these were described qualitatively in the results section, the missing items were left as is and the number of responses were shown in results.

\section{RESULTS}

Five-hundred and eleven participants completed the online survey. Three participants were excluded because they reported their age under 18 . Seven responses were excluded because they were duplicate IP addresses. A total of 500 participants completed the survey and are included in the analysis (mean age $39 \pm 15$; range 18-70; $68 \%$ female). Seventy-one percent of the participants $(n=353)$ screened were found to be positive for PTSD (mean score $17 \pm 6$ ). Seventy-six percent of the participants $(n=381)$ screened were found to be positive for depression (358 endorsed question one, 308 endorsed question two, and 285 endorsed both). Sixty-five percent of the participants $(n=326)$ screened were found to be positive for both PTSD and Depression. Mean values and standard deviations for the personality factors were as follows: extraversion (3.6 \pm 1.7$)$, agreeableness $(4.9 \pm 1.4)$, conscientiousness $(4.7 \pm 1.7)$, emotional stability $(3.9 \pm 1.6)$, and openness to experience $(5.1 \pm 1.6)$. These values detailed by first choice format preference are listed in Table 1. There were no differences between first choice format preference groups in participant characteristics except for age. Participant's age was significant, being driven by those choosing individual being younger than those choosing internet $\left(X^{2}=4.0 p=0.05\right)$ and those choosing group $\left(X^{2}=7.2 p=0.007\right)$. There was no difference in age between those choosing internet and group $\left(X^{2}=1.1 p=0.29\right)$.

Table 2. Respondents interest in each format and first and last choice format.

\begin{tabular}{|l|c|c|c|}
\hline & Internet & Individual & Group \\
\hline \hline Would refuse to take this version & $8(2 \%)$ & $10(2 \%)$ & $55(11 \%)$ \\
\hline Doesn't interest me & $43(9 \%)$ & $48(10 \%)$ & $107(22 \%)$ \\
\hline Neutral & $89(18 \%)$ & $71(14 \%)$ & $86(17 \%)$ \\
\hline Interests me & $188(38 \%)$ & $230(46 \%)$ & $149(30 \%)$ \\
\hline Very interested in this version & $168(34 \%)$ & $138(28 \%)$ & $96(20 \%)$ \\
\hline
\end{tabular}

Responders to "How interested would you be in the following formats?" (Internet $\mathrm{n}=496$; Individual $\mathrm{n}=497$; Group $\mathrm{n}=493$ ).

Participants were more interested (endorsing Interests me and Very Interested in $)$ in the Internet $(\mathrm{n}=356)$ and individual formats $(n=384)$ than the group format $(n=245)$. Fifty-five participants $(11 \%)$ refused a group format (Table 2). The distribution of interest was different between the three formats $\left(X^{2}=135.9, p<0.0001\right)$, between Internet and Individual $\left(X^{2}=9.7, p=0.05\right)$, between Internet and Group $\left(X^{2}=86.6, p<0.0001\right)$, and between Individual and Group $\left.X^{2}=79.9, p<0.0001\right)$.

Internet was rated as the first choice format for most participants (Internet 212 (43\%), Individual 187 (38\%),
Group 97 (20\%); First Choice $=496$ participants; Fig. 1). Group was the last choice for most participants (Internet $140(29 \%)$, Individual 70 (14\%), Group 279 (57\%); Last Choice $=489$ participants).

Table 3. Relative risk (95\% Confidence Interval) of first choice format.

\begin{tabular}{|l|c|c|}
\hline & Internet & Individual \\
\hline \hline Age & $0.99[0.98-1.01] ; p=0.33$ & $0.98[0.96-0.99] ; p=0.01$ \\
\hline Extraversion & $0.85[0.74-0.99] ; p=0.03$ & $0.86[0.75-0.99] ; p=0.05$ \\
\hline Emotional Stability & $1.18[1.01-1.37] ; p=0.03$ & $1.20[1.03-1.42] ; p=0.02$ \\
\hline
\end{tabular}

A multinomial logistic regression was conducted of first choice format to ascertain predictors. Only Age, Extraversion and Emotional Stability remained in the regression model. Group format was the reference category, thus the relative risk of Group was 1.0 and all relative risk values are relative to the group format.

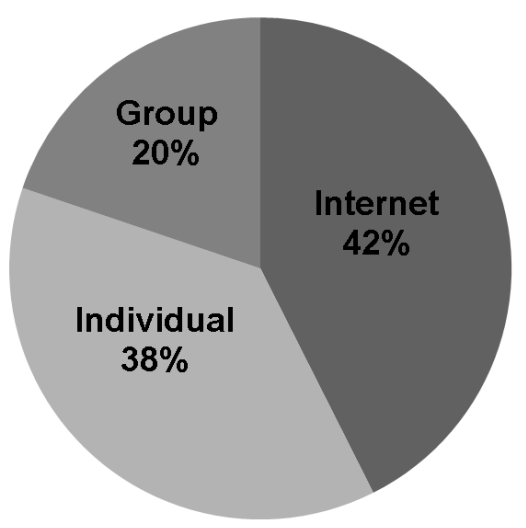

Fig. (1). First choice format.

The multinomial logistic regression of first choice format revealed age, extraversion, and emotional stability as significant predictors in the model, while the other predictors were not significant (Table 3). Given one unit increase in extraversion, the relative risk of choosing internet would be 0.85 times less likely and choosing individual would be 0.86 times less likely than group when the other variables in the model are held constant. Given one unit increase in emotional stability, the relative risk of choosing internet would be 1.18 times more likely and choosing individual would be 1.20 times more likely than group when the other variables in the model are held constant. Participants with higher emotional stability scores would be more likely to prefer internet or individual than the group format than people with lower emotional stability scores. Given a 10 year increase in age, the relative risk of choosing individual would be 0.90 times less likely than group when the other variables in the model are held constant. In a post-hoc binary logistic regression including only those who choose internet and individual formats, only age was significant where for every decade increase in age the relative risk of choosing individual was 0.90 times less likely than internet when the other variables in the model are held constant. Thus, younger participants were more likely to choose individual over internet. There were no significant predictors for last choice format.

Two-hundred and fifty-four (51\%) participants entered text into the optional descriptive field of Question 1. Pro and 
con responses are displayed in Table 4. Qualitative themes for the internet format included convenience, privacy, not having to share with others, and scheduling flexibility (Pro94, Con-42). The most prevalent pro response for the internet format was that it was convenient and easier. The most prevalent con response for the internet format was that there would be less accountability since it was self-directed. Qualitative themes for the individual format included developing a personal relationship with therapist, being guided more directly, privacy, and getting feedback (Pro-97, Con-27). The most prevalent pro response for the individual format was liking that format better followed by having personal and expert instruction. The most prevalent con response for the individual format was accessibility. Qualitative themes for the group format included being able to learn a new skill with other people, and comfort and motivation from other members (Pro-82, Con-79). The most prevalent pro response for the group format was the perceived benefit of working in a group. The most prevalent con responses for the group format were anxiety from social situations and negative group dynamics.

\section{DISCUSSION}

This cross-sectional study collected format preferences for an MMI from an online convenience sample of 500 participants and evaluated the relationship between the format preferences and age, gender, PTSD symptoms, depression status, and personality characteristics. Internet and individual were the preferred formats over group for most participants, reflected by their interest in the formats and the formats chosen for first and last choice. Age, extraversion and emotional stability were significant predictors in determining who may be more likely to choose one format over another.

Table 4. Counts of qualitative themes.

\begin{tabular}{|c|c|c|c|c|}
\hline Format & Pro & $\#$ & Con & $\#$ \\
\hline \multirow{8}{*}{ Group $n=161$ total comments) } & I like... & 11 & I don't like & 12 \\
\hline & Accountability & 8 & Accessibility/travel logistics & 13 \\
\hline & Benefits of group work & 30 & Avoidance of disclosure & 13 \\
\hline & Getting qs answered & 3 & Confidentiality breach & 6 \\
\hline & Interacting with people & 17 & Easier to hide & 0 \\
\hline & Motivation & 7 & Negative group dynamics & 17 \\
\hline & Understand material better & 6 & Social anxiety & 18 \\
\hline & Total & 82 & Total & 79 \\
\hline \multirow{9}{*}{ Individual ( $n=124$ total comments) } & I like... & 26 & I don't like... & 6 \\
\hline & Accountability & 8 & Accessibility/travel logistics & 12 \\
\hline & Getting questions answered & 2 & Confidentiality breach & 3 \\
\hline & Individualized & 6 & Too intimating & 6 \\
\hline & Interacting with people & 8 & & \\
\hline & Motivation & 6 & & \\
\hline & Personal/expert instruction & 25 & & \\
\hline & Understand material better & 16 & & \\
\hline & Total & 97 & Total & 27 \\
\hline \multirow{9}{*}{ Internet ( $n=136$ total comments) } & I like... & 9 & I don't like... & 10 \\
\hline & Confidential/Private/Anonymous & 12 & Accountability/Adherence/Motivation & 19 \\
\hline & Understand material better & 6 & Confidentiality Breach & 1 \\
\hline & Convenience/Easier & 67 & Impersonal/isolated & 9 \\
\hline & No Travel or costs $(\mathrm{n}=15)$ & & Limited Education & 3 \\
\hline & Self-paced/directed $(\mathrm{n}=14)$ & & & \\
\hline & Time/Scheduling Ease $(\mathrm{n}=21)$ & & & \\
\hline & Not specified why $(\mathrm{n}=17)$ & & & \\
\hline & Total & 94 & Total & 42 \\
\hline All options are good & & 33 & & \\
\hline
\end{tabular}

Counts for each theme are displayed. Participants could make comments on multiple formats, thus the number of comments exceeds the number of participants who responded to the text box on why they rated each format as they did. 
The participants in this study were mostly younger although there was quite a wide age range. Fifty percent of the participants were under 36 years of age. These demographics reflect the age distributions of national internet use averages [41]. There were $18 \%$ more women who responded to the survey than men. National averages show that slightly more women than men access the internet although women access more social media sites than men [42]. Thus, our participants reflect national averages of people who are on the internet. It is also reflective of US citizens whose median ages are 37.2 years [43].

The prevalence of participants who screened positive for depression $(76 \%)$, PTSD $(71 \%)$, or both $(65 \%)$ is higher in our sample than the reported prevalence of depression and PTSD in the general adult population. The prevalence of major depressive disorders and PTSD in the United States is estimated to be $6.7 \%$ and $3.5 \%$ respectively [44]. This is likely because our recruitment efforts included targeted advertising for people who may have depression and PTSD symptoms and past participants who were part of a PTSD study. Interestingly, a recent report on internet usage and depression found that $30 \%$ of college student internet users also exhibited depressive symptoms [45]. Another study similarly found a significant correlation between excessive internet use and depression but in a wider age range of participants (16-51 years old) [46]. While recruitment for this study was conducted through online advertisements, the survey did not include questions about frequency, duration, or type of internet use and thus, the internet usage patterns of the sample and whether they are excessive or not are unknown. It may be that people who use the internet have higher rates of depression and PTSD. However, to our knowledge, a large-scale study of internet use and depression and PTSD has not been conducted on the United States general population. Our sample also had a high prevalence of co-morbid PTSD and depression status similar to but higher than national averages. Nationally, depression and PTSD comorbidity rates are estimated to be approximately $36 \%$ [47]. Again, this higher prevalence in our sample is likely a result of oversampling in these populations. The survey used brief screening tools to evaluate PTSD symptoms, PTSD status and depression status. While these brief self-report instruments have been validated against gold-standard screening instruments, they cannot replace clinical interview and definitive diagnosis of major psychiatric disorders. The higher prevalence of depression and PTSD in our sample limits the generalizability of these results to the general population of the United States.

It is uncertain how this higher prevalence of self-report depression and PTSD affected our results. One would expect that people with depression and PTSD symptoms would prefer internet and individual format options for MMI as observed for other interventions [23]. However, in the multinomial regression analysis PTSD symptom score and depression status were not predictors for format choice. It may be that age and personality are stronger predictors than PTSD and depression and supersede any effect of PTSD and depression symptoms status. For example, a depressed person who is also more extraverted would prefer a group format over internet or individual regardless of their depression status. This could be true for PTSD symptoms as well. While we hypothesized that those with PTSD would prefer internet and individual over group because of aversion to group sharing and desire to avoid triggers, age and personality may override the PTSD symptoms in the type of format they would prefer. For example, it may be that people with PTSD who agree to group therapy have higher extraversion and emotional stability scores than most people with PTSD who would refuse group therapy. Future studies should examine the interactions between PTSD and depression and personality traits and differential responses to different formats.

Our sample personality factors were within the standard deviation of the means of a normative database of 1813 males and female participants of all ethnicities [36]. For example, the mean value for extraversion in our sample was $3.6 \pm 1.7$ and the normative database mean value was 4.44 \pm 1.45 . The other traits were also comparable; agreeableness (Survey $4.9 \pm 1.4$; Norm $5.2 \pm 1.1$ ) conscientiousness (Survey $4.7 \pm 1.7$; Norm $5.4 \pm 1.3$ ), emotional stability (Survey $3.9 \pm 1.6$; Norm $4.8 \pm 1.4$ ), and openness to experience (Survey $5.1 \pm 1.6$; Norm $5.4 \pm 1.1$ ) scores for our sample were all within one standard deviation of normative scores [36]. There is a link between PTSD and low extraversion and emotional stability, while there are no conclusive relationships between the other traits and PTSD [48]. The mean values for our sample were slightly lower than the normative values perhaps reflecting the higher prevalence of PTSD symptoms, however they were still within normal ranges.

Considering the limited demographics collected, our sample was representative on age, had a greater number of women, and similar mean values of personality characteristics compared to the general population. The higher prevalence of self-report PTSD and depression symptoms most likely relates to the recruitment methodology. Keeping this in mind, our participants were more interested in the alternative delivery formats of MMI. This was reflected in two ways. First, participants were more interested in internet and individual formats compared to group formats. In fact, $55(11 \%)$ participants stated they would refuse a group format compared to only $8(2 \%)$ for the internet format and $10(2 \%)$ for the individual format. Since the standard administration of MMI's is a group format, this highlights the need to develop alternative delivery formats. Additionally, participants were slightly more interested in the individual format compared to the internet format. This may reflect a desire for greater privacy in sharing and yet more guidance and support than the internet format would provide. This was supported by the statements made in the text option. Second, interest in alternative formats was reflected in the first choice and last choice preferences. Internet was the first choice of most participants (43\%) followed closely by the individual format (38\%) and lastly the group format with only $20 \%$ endorsement. The last choice mirrored this result with Group being the last choice for most participants $(57 \%)$ followed by the internet format $(29 \%)$ and finally the individual format (14\%). Again, this may reflect more privacy, increased flexibility with scheduling and more guidance and support for the intervention as seen described in the text answers.

Internet and individual formats offer different benefits. Internet formats, in the way we described it for this study 
(visiting a website from any location and reviewing the material online), would allow for greater anonymity and privacy, reduced travel, and scheduling flexibility. It would not provide person to person interaction or discussion about the material. The internet format could be viewed as low dose mindfulness meditation therapy because of the lack of teacher guidance and interaction. This would not be ideal for people with severe mental health illness who would require more sensitive and immediate care. Workplace programs and general stress reduction programs could be well-suited to internet delivery formats. Individual or one-on-one therapies would also allow for greater anonymity and privacy and scheduling flexibility compared to the group format but the participant/patient would still need to travel to visit the therapist. This option would be beneficial for those with more severe symptomology and the need for a higher dose of mindfulness meditation therapy. Currently, most MMI's are administered in the group format. The greatest pros people reported to the group format were the benefits of group work and interacting with others. Clinicians could take into account the pros and cons of each format and the qualities of each patient when referring for this type of intervention. For example, a patient with social anxiety may be more comfortable in a one-on-one or internet format whereas someone who is very social and lives near group class would do fine in a group format class. In addition to using the results of this study to help inform clinical recommendations of format type, these results highlight the need for more research and implementation of alternate delivery methods of MMI's, especially considering the $11 \%$ refusal of the group format.

Our multinomial logistic regression analysis identified age, extraversion, and emotional stability as significant predictors of first choice format. We had hypothesized that extraversion would predict first choice format with participants with higher extraversion being more likely to choose group over internet or individual. While this finding seems intuitive, to our knowledge this is the first time it has been formally evaluated in this context. We had hypothesized that younger participants would be more likely to choose internet compared to group. However, younger participants in our sample were also more likely to choose individual over group. The fact that internet users were sampled in this study may have influenced any age bias in choosing internet over group. Younger people in other studies have also shown preference to individual therapies over group therapies [19]. Participants with higher emotional stability scores were more likely to choose internet and individual formats than the group format. Gender, agreeableness, conscientiousness, and openness to experience were not found to be significant predictors of first choice format and may not be important in alternative format choice. Surprisingly, no predictors were significant for the last choice format choice. We had anticipated that personality may influence aversion as well as preference to a particular format but this was not the case in our study. Future research examining predictors for format choice will support the research and implementation of these alternative formats of MMI's. Research study designs may include pragmatic trials or even patient choice designs to more thoroughly evaluate the interactions between participant/patient characteristics and effect of MMI's [49]. Perhaps more importantly, it may also allow for greater individualization of health care extending the precision medicine pharmacologic approach to mind-body medicine $[50,51]$

There are a number of limitations to this study that should be taken into account when interpreting the results. The study sample is not a population-based survey. Survey participants were people who use the internet and the survey was administered online and thus, there was an inherent bias towards the internet format. Recruitment was targeted to those with PTSD and/or depression. Targeted recruitment may exclude those with significant disadvantaging circumstances (i.e. socioeconomic status, elderly individuals, and individuals with a mental illness). The demographics collected in the survey were very limited. Other demographic factors such as education, socioeconomic status, ethnicity, and location may play a role in predicting format delivery type and should be included in future analyses. Taken together, caution should be exercised when generalizing these results to the general population.

\section{CONCLUSION}

Internet and individual MMI formats were preferred to the standard group format. In fact, $11 \%$ of the participants said they would refuse the group format. Since most MMI's are currently taught in the group format, this warrants increased research and implementation of alternative delivery formats including internet and individual formats. Age, extraversion, and emotional stability were significant predictors in determining who would choose internet or individual over the group format. While understanding these predictors is helpful, further research into other predictors not included in this study will be valuable especially in individualized MMI's for participants and patients who would benefit from them.

\section{CONFLICT OF INTEREST}

The authors confirm that this article content has no conflict of interest.

\section{ACKNOWLEDGEMENTS}

The authors would like to thank Julien Cayton for his help with this project. This work was supported by the National Center for Complementary and Alternative Medicine of the National Institutes of Health [Grant numbers K01AT004951, K24AT005121]. The content of this paper is solely the responsibility of the authors and does not necessarily represent the official views of the National Institutes of Health.

\section{REFERENCES}

[1] Khoury B, Lecomte T, Fortin G, et al. Mindfulness-based therapy: a comprehensive meta-analysis. Clin Psychol Rev 2013; 33(6): 763-71. 
[2] Hofmann SG, Sawyer AT, Witt AA, Oh D. The effect of mindfulness-based therapy on anxiety and depression: A metaanalytic review. J Consult Clin Psychol 2010; 78(2): 169-83.

[3] Keng SL, Smoski MJ, Robins CJ. Effects of mindfulness on psychological health: a review of empirical studies. Clin Psychol Rev 2011; 31(6): 1041-56.

[4] Chiesa A, Serretti A. Mindfulness-based stress reduction for stress management in healthy people: a review and meta-analysis. J Altern Complement Med 2009; 15(5): 593-600.

[5] Chiesa A, Serretti A. Mindfulness based cognitive therapy for psychiatric disorders: a systematic review and meta-analysis. Psychiatry Res 2011; 187(3): 441-53.

[6] Chiesa A, Serretti A. Mindfulness-based interventions for chronic pain: a systematic review of the evidence. J Altern Complement Med 2011; 17(1): 83-93.

[7] Grossman P, Niemann L, Schmidt S, Walach H. Mindfulness-based stress reduction and health benefits. A meta-analysis. J Psychosom Res 2004; 57(1): 35-43.

[8] Vollestad J, Nielsen MB, Nielsen GH. Mindfulness- and acceptance-based interventions for anxiety disorders: a systematic review and meta-analysis. Br J Clin Psychol 2012; 51(3): 239-60.

[9] Wahbeh H, Elsas SM, Oken BS. Mind-body interventions: applications in neurology. Neurology 2008; 70(24): 2321-8.

[10] Wahbeh H, Lane JB, Goodrich E, Miller M, Oken BS. One-on-one mindfulness meditation trainings in a research setting. Mindfulness 2014; 5(1): 88-99.

[11] Tacon AM, McComb J, Caldera Y, Randolph P. Mindfulness meditation, anxiety reduction, and heart disease: a pilot study. Fam Community Health 2003; 26(1): 25-33.

[12] Kabat-Zinn J, Massion AO, Kristeller J, et al. Effectiveness of a meditation-based stress reduction program in the treatment of anxiety disorders. Am J Psychiatry 1992; 149(7): 936-43.

[13] Carlson LE, Garland SN. Impact of mindfulness-based stress reduction (MBSR) on sleep, mood, stress and fatigue symptoms in cancer outpatients. Int J Behav Med 2005; 12(4): 278-85.

[14] Carlson LE, Speca M, Faris P, Patel KD. One year pre-post intervention follow-up of psychological, immune, endocrine and blood pressure outcomes of mindfulness-based stress reduction (MBSR) in breast and prostate cancer outpatients. Brain Behav Immun 2007; 21(8): 1038-49.

[15] Price CJ, McBride B, Hyerle L, Kivlahan DR. Mindful awareness in body-oriented therapy for female veterans with post-traumatic stress disorder taking prescription analgesics for chronic pain: a feasibility study. Altern Ther Health Med 2007; 13(6): 32-40.

[16] Allen NB, Chambers R, Knight W. Mindfulness-based psychotherapies: a review of conceptual foundations, empirical evidence and practical considerations. Aust N Z J Psychiatry 2006; 40(4): 285-94

[17] Wahbeh H. Mindfulness meditation for posttraumatic stress disorder. In: Ie A, Ngnoumen CT, Langer E, Eds. The Wiley Blackwell Handbook of Mindfulness. $1^{\text {st }}$ ed. Chichester, UK: John Wiley \& Sons Ltd 2014; pp. 776-93.

[18] Wahbeh H, Senders A, Neuendorf R, Cayton J. Complementary and alternative medicine for posttraumatic stress disorder symptoms: a systematic review. J Evid Based Complementary Altern Med 2014; 19(3): 163 -77

[19] Kracen AC, Mastnak JM, Loaiza KA, Matthieu MM. Group therapy among $\mathrm{OEF} / \mathrm{OIF}$ veterans: treatment barriers and preferences. Mil Med 2013; 178(1): e146-9.

[20] Kearney DJ, McDermott K, Malte C, Martinez M, Simpson TL. Association of participation in a mindfulness program with measures of PTSD, depression and quality of life in a veteran sample. J Clin Psychol 2012; 68(1): 101-16.

[21] Kuyken W, Byford S, Taylor RS, et al. Mindfulness-based cognitive therapy to prevent relapse in recurrent depression. J Consult Clin Psychol 2008; 76(6): 966-78.

[22] Lau MA, Yu AR. New developments in research on mindfulnessbased treatments: introduction to the special issue. J Cognitive Psychother 2009; 23(3): 179-84.

[23] Barak A, Hen L, Boniel-Nissim M, Shapira N. A comprehensive review and a meta-analysis of the effectiveness of Internet-based psychotherapeutic interventions. J Technol Human Services 2008; 26(2-4): 109-60.
[24] Meyer B, Berger T, Caspar F, Beevers CG, Andersson G, Weiss M. Effectiveness of a novel integrative online treatment for depression (Deprexis): randomized controlled trial. J Med Internet Res 2009; 11(2): e15.

[25] Thompson NJ, Walker ER, Obolensky N, et al. Distance delivery of mindfulness-based cognitive therapy for depression: project UPLIFT. Epilepsy Behav 2010; 19(3): 247-54.

[26] Ljotsson B, Falk L, Vesterlund AW, et al. Internet-delivered exposure and mindfulness based therapy for irritable bowel syndrome--a randomized controlled trial. Behav Res Ther 2010; 48(6): 531-9.

[27] Ljotsson B, Hedman E, Lindfors P, et al. Long-term follow-up of internet-delivered exposure and mindfulness based treatment for irritable bowel syndrome. Behav Res Ther 2011; 49(1): 58-61.

[28] Morledge TJ, Allexandre D, Fox E, et al. Feasibility of an online mindfulness program for stress management--a randomized, controlled trial. Ann Behav Med 2013; 46(2): 137-48.

[29] Gardner-Nix J, Backman S, Barbati J, Grummitt J. Evaluating distance education of a mindfulness-based meditation programme for chronic pain management. J Telemed Telecare 2008; 14(2): 8892 .

[30] Wolever RQ, Bobinet KJ, McCabe K, et al. Effective and viable mind-body stress reduction in the workplace: a randomized controlled trial. J Occup Health Psychol 2012; 17(2): 246-58.

[31] Gluck TM, Maercker A. A randomized controlled pilot study of a brief web-based mindfulness training. BMC Psychiatry 2011; 11: 175.

[32] de Lisle SM, Dowling NA, Allen JS. Mindfulness-based cognitive therapy for problem gambling. Clin Case Stud 2011; 10(3): 210-28

[33] Cavanagh K, Strauss C, Forder L, Jones F. Can mindfulness and acceptance be learnt by self-help?: A systematic review and metaanalysis of mindfulness and acceptance-based self-help interventions. Clin Psychol Rev 2014; 34(2): 118-29.

[34] Lang AJ, Stein MB. An abbreviated PTSD checklist for use as a screening instrument in primary care. Behav Res Ther 2005; 43(5): 585-94

[35] Whooley MA, Avins AL, Miranda J, Browner WS. Case-finding instruments for depression. Two questions are as good as many. J Gen Intern Med 1997; 12(7): 439-45.

[36] Gosling SD, Rentfrow PJ, Swann WB. A very brief measure of the Big-Five personality domains. J Res Personality 2003; 37: 504-28.

[37] Braun V, Clarke V. Using thematic analysis in psychology. Qual Res Psychol 2006; 3(2): 77-101.

[38] Saldana J. The Coding Manual for Qualitative Research. Lo Angeles: SAGE 2013.

[39] Blanchard EB, Jones-Alexander J, Buckley TC, Forneris CA. Psychometric properties of the PTSD Checklist (PCL). Behav Res Ther 1996; 34(8): 669-73.

[40] Digman JM. Personality structure: emergence of the five factor model. Annu Rev Psychol 1990; 41: 417-40.

[41] File T. Computer and Internet Use in the United States. Washington, DC: U.S. Census Beurau 2013.

[42] Fallows D. How women and men use the internet. Pew Research; 2008; Available from: http://www.pewinternet.org/2005/12/28/ how-women-and-men-use-the-internet/.

[43] Howden LM, Meyer JA. Age and Sex Composition: 2010 Washington, DC 2011; Available from: http//www.census.gov/p $\mathrm{rod} / \mathrm{cen} 2010 / \mathrm{briefs} / \mathrm{c} 2010 \mathrm{br}-03 . p d f$.

[44] Kessler RC, Chiu WT, Demler O, Merikangas KR, Walters EE. Prevalence, severity, and comorbidity of 12-month DSM-IV disorders in the National Comorbidity Survey Replication. Arch Gen Psychiatry 2005; 62(6): 617-27.

[45] Katikalapudi R, Chellappan S, Montgomery F, Wunsch D, Lutzen $\mathrm{K}$. Associating internet usage with depressive behavior among college students. Technol Society Magazine IEEE 2012; 31(4): 7380 .

[46] Morrison CM, Gore H. The relationship between excessive internet use and depression: a questionnaire-based study of 1,319 young people and adults. Psychopathology 2010; 43(2): 121-6.

[47] Campbell DG, Felker BL, Liu CF, et al. Prevalence of depressionPTSD comorbidity: implications for clinical practice guidelines and primary care-based interventions. J Gen Intern Med 2007; 22(6): $711-8$ 
[48] Jaksic N, Brajkovic L, Ivezic E, Topic R, Jakovljevic M. The role of personality traits in posttraumatic stress disorder (PTSD). Psychiatr Danub 2012; 24(3): 256-66.

[49] Patsopoulos NA. A pragmatic view on pragmatic trials. Dialogues Clin Neurosci 2011; 13(2): 217-24.
[50] Hatz MH, Schremser K, Rogowski WH. Is individualized medicine more cost-effective? A systematic review. Pharmacoeconomics 2014; 32(5): 443-55.

[51] Chae H, Lyoo IK, Lee SJ, et al. An alternative way to individualized medicine: psychological and physical traits of Sasang typology. J Altern Complement Med 2003; 9(4): 519-28.

(C) Wahbeh et al.; Licensee Bentham Open.

This is an open access article licensed under the terms of the Creative Commons Attribution Non-Commercial License (http://creativecommons.org/licenses/by$\mathrm{nc} / 3.0 /$ ) which permits unrestricted, non-commercial use, distribution and reproduction in any medium, provided the work is properly cited. 